\title{
Equivalence of Kuo and Thom quantities for analytic functions
}

\author{
By Karim BeKKA*) and Satoshi KoIKE**) \\ (Communicated by Kenji FukAYA, M.J.A., Feb. 12, 2021)
}

\begin{abstract}
Sufficiency of jets is a very important notion introduced by René Thom in order to establish the structural stability theory. The criteria for some sufficiency of jets are known as the Kuo condition and Thom type inequality, which are defined using the Kuo quantity and Thom quantity. Therefore these quantities are meaningful. In this paper we show the equivalence of Kuo and Thom quantities. Then we apply this result to the relative conditions to a given closed set.
\end{abstract}

Key words: Kuo condition; Thom type inequality; Kuo quantity; Thom quantity; sufficiency of jet.

1. Introduction. Let $f:\left(\mathbf{R}^{n}, 0\right) \rightarrow(\mathbf{R}, 0)$ be a $C^{r}$ function germ. The $r$-jet of $f$ at $0 \in \mathbf{R}^{n}, j^{r} f(0)$, has a unique polynomial representative $z$ of degree not exceeding $r$. We do not distinguish the $r$-jet $j^{r} f(0)$ and the polynomial representative $z$ here.

Kuiper-Kuo condition. There is a positive number $C>0$ such that

$$
\|\operatorname{grad} f(x)\| \geq C\|x\|^{r-1}
$$

holds in some neighbourhood of $0 \in \mathbf{R}^{n}$.

Note that the Kuiper-Kuo condition depends only of its $r$-jet $z=j^{r} f(0)$, and it is independent of the choice of representative.

The Kuiper-Kuo condition is well-known as a criterion for $C^{0}$-sufficiency and $V$-sufficiency of $z$ in $C^{r}$ functions (N. Kuiper [6], T. C. Kuo [7], J. Bochnak and S. Łojasiewicz [4]). See $\S 2$ for the definitions of $C^{0}$-sufficiency and $V$-sufficiency of jet.

Kuo condition. There are positive numbers $C, \alpha, \bar{w}>0$ such that

$$
\|\operatorname{grad} f(x)\| \geq C\|x\|^{r-1} \text { in } \mathcal{H}_{r}(f ; \bar{w}) \cap\{\|x\|<\alpha\},
$$

where $\mathcal{H}_{r}(f ; \bar{w}):=\left\{x \in \mathbf{R}^{n}:|f(x)| \leq \bar{w}\|x\|^{r}\right\}$ is the horn-neighbourhood of $f^{-1}(0)$ of degree $r$ and width $\bar{w}($ T. C. Kuo [8]).

Note that this Kuo condition is also a condition on the $r$-jet $z=j^{r} f(0)$, and it is independent of the representative $f$.

2010 Mathematics Subject Classification. Primary 57R45; Secondary $58 \mathrm{~K} 40$.

*) Institut de Recherche Mathématique de Rennes, Université de Rennes 1, Campus Beaulieu, 35042 Rennes cedex, France.

**) Department of Mathematics, Hyogo University of Teacher Education, Kato, Hyogo 673-1494, Japan.
The Kuo condition is a criterion for $V$-sufficiency of $z$ in $C^{r}$ functions.

Condition $(\widetilde{\boldsymbol{K}})$. There is a positive number $C>0$ such that

$$
\|x\|\|\operatorname{grad} f(x)\|+|f(x)| \geq C\|x\|^{r}
$$

holds in some neighbourhood of $0 \in \mathbf{R}^{n}$.

This condition is the Kuo condition in a different way. Therefore condition $(\widetilde{K})$ is also a criterion for $V$-sufficiency of $z$ in $C^{r}$ functions.

On the other hand, R. Thom formulated the following condition as a sufficient condition for $z$ to be $C^{0}$-sufficient in $C^{r}$-functions.

Thom type inequality. There are positive numbers $K, \beta>0$ such that

$\sum_{i<j}\left|x_{i} \frac{\partial f}{\partial x_{j}}-x_{j} \frac{\partial f}{\partial x_{i}}\right|^{2}+|f(x)|^{2} \geq K\|x\|^{2 r}$ for $\|x\|<\beta$.

It is shown in [1] that Thom type inequality condition is equivalent to the Kuiper-Kuo condition.

Throughout this paper, we denote by $\mathbf{N}$ the set of natural numbers in the sense of positive integers. Let $s \in \mathbf{N} \cup\{\infty, \omega\}$, and let $\mathcal{E}_{[s]}(n, p)$ denote the set of $C^{s}$ map-germs: $\left(\mathbf{R}^{n}, 0\right) \rightarrow\left(\mathbf{R}^{p}, 0\right)$.

Now we introduce the Kuo quantity $K_{m}$ and Thom quantity $T_{m}$. The Thom quantity is a generalisation of the left side of Thom type inequality, and the Kuo quantity is a generalisation of the left side of a condition equivalent to condition $(\widetilde{K})$.

Definition 1.1. Let $f \in \mathcal{E}_{[s]}(n, p), n \geq p$, and let $m \in \mathbf{N}$. Let us define two functions of the variable $x$ : 
(1.1) $K_{m}(f, x)$

$$
\begin{aligned}
& :=\|x\|^{m} \sum_{1 \leq i_{1}<\ldots<i_{p} \leq n}\left|\operatorname{det}\left(\frac{D\left(f_{1}, \ldots, f_{p}\right)}{D\left(x_{i_{1}}, \ldots, x_{i_{p}}\right)}(x)\right)\right|^{m}+\|f(x)\|^{m}, \\
& T_{m}(f, x) \\
& :=\sum_{1 \leq i_{1}<\ldots<i_{p+1} \leq n}\left|\operatorname{det}\left(\frac{D\left(f_{1}, \ldots, f_{p}, \rho\right)}{D\left(x_{i_{1}}, \ldots, x_{i_{p+1}}\right)}(x)\right)\right|^{m}+\|f(x)\|^{m},
\end{aligned}
$$$$
\text { (1.2) } T_{m}(f, x)
$$

where $\rho(x)=\|x\|^{2}$. Note that $T_{m}(f, x)=\|f(x)\|^{m}$ in the case where $n=p$.

Related to the Kuo condition and Thom type inequality, we have shown the following result.

Theorem 1.2. ([1], Theorem 2.4). Let $r \in$ N. For $f \in \mathcal{E}_{[r]}(n, p), n \geq p$, the following conditions are equivalent.

(1) There are positive numbers $C, \alpha>0$ such that $K_{2}(f, x) \geq C\|x\|^{2 r}$ for $\|x\|<\alpha$.

(2) There are positive numbers $K, \beta>0$ such that $T_{2}(f, x) \geq K\|x\|^{2 r}$ for $\|x\|<\beta$.

The main purpose of this paper is to show the equivalence of the Kuo quantity and Thom quantity.

Theorem 1.3. (Main Theorem). Let $f \in$ $\mathcal{E}_{[\omega]}(n, p), n \geq p$. Then for any $m \in \mathbf{N}$,

$$
K_{m}(f, .) \approx T_{m}(f, .) .
$$

The main result is a generalisation of Theorem 1.2 in the sense that it follows from Theorem 1.3 and the notion of $r$-compatibility (cf. Definition 4.1 or [1]).

Throughout this paper, we use the equivalence $\approx$ in the following sense:

Let $f, g: U \rightarrow \mathbf{R}$ be non-negative functions, where $U \subset \mathbf{R}^{N}$ is an open neighbourhood of $0 \in$ $\mathbf{R}^{N}$. If there are real numbers $K>0, \delta>0$ with $B_{\delta}(0) \subset U$ such that $f(x) \leq K g(x)$ for any $x \in B_{\delta}(0)$, where $B_{\delta}(0)$ is a closed ball in $\mathbf{R}^{N}$ of radius $\delta$ centred at $0 \in \mathbf{R}^{N}$, then we write $f \precsim g$ (or $g \succsim f$ ). If $f \precsim g$ and $f \succsim g$, we write $f \approx g$.

In the next section we mention the definitions of $C^{0}$-sufficiency and $V$-sufficiency of jets, and give the notion of the relative jet of a $C^{s}$ mapping to a given closed set $\Sigma$. We shall show our Main Theorem in $\$ 3$, and apply the theorem to the relative conditions to a closed set $\Sigma$ in $\S 4$.

\section{Preliminaries.}

2.1. Sufficiency of jets. Let $s \in \mathbf{N} \cup\{\infty, \omega\}$. Let us recall $\mathcal{E}_{[s]}(n, p)$, the set of $C^{s}$ map-germs: $\left(\mathbf{R}^{n}, 0\right) \rightarrow\left(\mathbf{R}^{p}, 0\right)$. Let $j^{r} f(0)$ denote the $r$-jet $(r \in$ $\mathbf{N})$ of $f$ at $0 \in \mathbf{R}^{n}$ for $f \in \mathcal{E}_{[s]}(n, p), s \geq r$, and let $J^{r}(n, p)$ denote the set of $r$-jets in $\mathcal{E}_{[s]}(n, p)$.
We say that $f, g \in \mathcal{E}_{[s]}(n, p)$ are $C^{0}$-equivalent (resp. $S V$-equivalent), if there exists a local homeomorphism $\sigma:\left(\mathbf{R}^{n}, 0\right) \rightarrow\left(\mathbf{R}^{n}, 0\right)$ such that $f=g \circ \sigma$ (resp. $\left.\sigma\left(f^{-1}(0)\right)=g^{-1}(0)\right)$. In addition, we say that $f, g \in \mathcal{E}_{[s]}(n, p)$ are $V$-equivalent, if $f^{-1}(0)$ is homeomorphic to $g^{-1}(0)$ as germs at $0 \in \mathbf{R}^{n}$.

Let $w \in J^{r}(n, p)$. We call the $r$-jet $w C^{0}$-sufficient, $S V$-sufficient and $V$-sufficient in $C^{s}$ mappings, $s \geq r$, if any two realisations $f, g \in \mathcal{E}_{[s]}(n, p)$ of $w$, namely $j^{r} f(0)=j^{r} g(0)=w$, are $C^{0}$-equivalent, $S V$-equivalent and $V$-equivalent, respectively.

Let us recall the Thom type inequality for $f \in \mathcal{E}_{[s]}(n, p), n \geq p$ :

There are positive numbers $K, \alpha, \beta>0$ such that $T_{2}(f, x) \geq K\|x\|^{\alpha}$ for $\|x\|<\beta$.

As mentioned in the Introduction, R. Thom considered this condition with $\alpha=2 r$ in the function case as a sufficient condition for $z=j^{r} f(0)$ to be $C^{0}$-sufficient in $C^{r}$ functions. On the other hand, he considered this condition in the mapping case as a sufficient condition for $S V$-sufficiency of jet.

On the other hand, the Kuo condition is a criterion for $V$-sufficiency of $z=j^{r} f(0)$ in $C^{r}$ functions. This condition is generalised to the mapping case, as a criterion for $V$-sufficiency of $z=$ $j^{r} f(0)$ in $C^{r}$ mappings: $\left(\mathbf{R}^{n}, 0\right) \rightarrow\left(\mathbf{R}^{p}, 0\right), n \geq p$. For the details, see T. C. Kuo [9].

2.2. Relative jet to a given closed set. Throughout this paper, let $\Sigma$ be a germ of a given closed subset of $\mathbf{R}^{n}$ at $0 \in \mathbf{R}^{n}$ such that $0 \in \Sigma$. Then we denote by $d(x, \Sigma)$ the distance from a point $x \in$ $\mathbf{R}^{n}$ to the subset $\Sigma$.

We consider on $\mathcal{E}_{[s]}(n, p)$ the following equivalence relation:

Two map-germs $f, g \in \mathcal{E}_{[s]}(n, p)$ are $r$ - $\Sigma$-equivalent, denoted by $f \sim g$, if there exists a neighbourhood $U$ of 0 in $\mathbf{R}^{n}$ such that the $r$-jet extensions of $f$ and $g$ satisfy $j^{r} f(\Sigma \cap U)=j^{r} g(\Sigma \cap U)$.

We denote by $j^{r} f(\Sigma ; 0)$ the equivalence class of $f$, and by $J_{\Sigma}^{r}(n, p)$ the quotient set $\mathcal{E}_{[s]}(n, p) / \sim$.

We can define the notions of $C^{0}$-sufficiency, $S V$-sufficiency and $V$-sufficiency of relative jets to $\Sigma$, similarly to in the non-relative case. In [2] we gave criteria for the relative $r$-jet to be $C^{0}$-sufficient and $V$-sufficient in $\mathcal{E}_{[r]}(n, p)$ or $\mathcal{E}_{[r+1]}(n, p)$, using the relative Kuiper-Kuo condition and relative Kuo condition (or condition $\left(\widetilde{K}_{\Sigma}\right)$ ), respectively.

3. Proof of Main Theorem. In this section we show the equivalence between the Kuo quantity $K_{m}$ and the Thom quantity $T_{m}$, namely Theo- 
rem 1.3. Before we give the proof, let us examine an example.

Example 3.1. Let $f=\left(f_{1}, f_{2}\right):\left(\mathbf{R}^{2}, 0\right) \rightarrow$ $\left(\mathbf{R}^{2}, 0\right)$ be a polynomial mapping defined by $f_{1}(x, y)=x-y^{2}, f_{2}(x, y)=x^{2}$. Then we have $f_{1}(x, y)^{2}+f_{2}(x, y)^{2}=\left(x-y^{2}\right)^{2}+x^{4}$, $\operatorname{det}\left(\frac{D\left(f_{1}, f_{2}\right)}{D(x, y)}((x, y))\right)=4 x y$.

Therefore we have

$$
\begin{aligned}
T_{2}(f,(x, y)) & =\left(x-y^{2}\right)^{2}+x^{4}, \\
K_{2}(f,(x, y)) & =16\left(x^{2}+y^{2}\right) x^{2} y^{2}+\left(x-y^{2}\right)^{2}+x^{4} .
\end{aligned}
$$

To show that $T_{2}(f,(x, y)) \approx K_{2}(f,(x, y))$, we consider two cases.

In the case where $\left|x-y^{2}\right| \leq \frac{1}{2} y^{2}$, we have $x \geq \frac{1}{2} y^{2}$. Therefore $64 x^{4} \geq 16 x^{2} y^{4}$ and since for any constant $C>65,16 x^{4} y^{2}=o\left((C-65) x^{4}\right)$ we get

$$
C T_{2}(f,(x, y)) \geq K_{2}(f,(x, y)) \geq T_{2}(f,(x, y))
$$

in a small neighbourhood of $(0,0) \in \mathbf{R}^{2}$. In the case where $\left|x-y^{2}\right| \geq \frac{1}{2} y^{2}$ we can see that

$$
\begin{aligned}
& \left(x-y^{2}\right)^{2}+x^{4} \geq \frac{1}{4} y^{4}+x^{4} \\
& \quad \geq 16 x^{2} y^{4}+16 x^{4} y^{4}=16\left(x^{2}+y^{2}\right) x^{2} y^{2}
\end{aligned}
$$

in a small neighbourhood of $(0,0) \in \mathbf{R}^{2}$. Thus, for any constant $C>65$, we have

$$
T_{2}(f,(x, y)) \leq K_{2}(f,(x, y)) \leq C T_{2}(f,(x, y))
$$

in a small neighbourhood of $(0,0) \in \mathbf{R}^{2}$, it follows that $T_{2}(f,(x, y)) \approx K_{2}(f,(x, y))$.

Let $\operatorname{ord}(\gamma(t))$ denote the order of $\gamma$ in $t$ for a $C^{\omega}$ function $\gamma:[0, \delta) \rightarrow \mathbf{R}$.

Proof of Theorem 1.3. It is obvious that $K_{m}(f,.) \succsim T_{m}(f,$.$) . Therefore we have to show the$ converse.

We first remark that if $x$ and $y$ are bigger than or equal to 0 , we have

$$
(x+y)^{m} \geq x^{m}+y^{m} \geq \frac{(x+y)^{m}}{2^{m}} .
$$

It follows that

$$
\begin{aligned}
& K_{m}(f, x) \approx v^{m}(x)+u^{m}(x) \approx(h(x))^{m}, \\
& T_{m}(f, x) \approx w^{m}(x)+u^{m}(x) \approx(g(x))^{m},
\end{aligned}
$$

where $u(x)=\|f(x)\|$,

$$
v(x)=\|x\| \sum_{1 \leq i_{1}<\ldots<i_{p} \leq n}\left|\operatorname{det}\left(\frac{D\left(f_{1}, \ldots, f_{p}\right)}{D\left(x_{i_{1}}, \ldots, x_{i_{p}}\right)}(x)\right)\right|,
$$

$$
w(x)=\sum_{1 \leq i_{1}<\ldots<i_{p+1} \leq n}\left|\operatorname{det}\left(\frac{D\left(f_{1}, \ldots, f_{p}, \rho\right)}{D\left(x_{i_{1}}, \ldots, x_{i_{p+1}}\right)}(x)\right)\right|,
$$

$\left(\right.$ where $\left.\rho(x)=\|x\|^{2}\right)$,

$h(x)=v(x)+u(x)$ and $g(x)=w(x)+u(x)$.

Suppose now that $K_{m}(f,.) \precsim T_{m}(f,$.$) does not$ hold. Then by the curve selection lemma, there is a $C^{\omega}$ curve $\tilde{\lambda}=(\lambda, C):[0, \delta) \rightarrow \mathbf{R}^{n} \times \mathbf{R}$ with $\tilde{\lambda}(0)=$ $(0,0)$ and $\tilde{\lambda}(t) \in\left(\mathbf{R}^{n} \backslash\{0\}\right) \times \mathbf{R}^{*}$, for $t \neq 0$, such that

$$
(C(t))^{m} K_{m}(f, \lambda(t))>T_{m}(f, \lambda(t)) .
$$

We may write (3.1) as:

$$
(C(t)(h \circ \lambda(t)))^{m}>(g \circ \lambda(t))^{m} .
$$

Here we remark that the functions $g \circ \lambda, h \circ \lambda, u \circ \lambda$, $v \circ \lambda$ and $w \circ \lambda$ are real analytic on $[0, \delta)$ and satisfying the conditions

$$
\begin{aligned}
g \circ \lambda(0) & =h \circ \lambda(0)=u \circ \lambda(0)=v \circ \lambda(0) \\
& =w \circ \lambda(0)=0
\end{aligned}
$$

and

$$
\lambda(t) \neq 0, C(t)>0, h \circ \lambda(t)>0, g \circ \lambda(t) \geq 0
$$

for $0<t<\delta$.

By (3.2), $C(t)(h \circ \lambda(t))>u \circ \lambda(t)$,

$C(t)(h \circ \lambda(t))>w \circ \lambda(t)$ and

$$
v \circ \lambda(t)=h \circ \lambda(t)-u \circ \lambda(t) \geq h \circ \lambda(t)(1-C(t)) .
$$

Then we have

$$
\left\{\begin{array}{l}
\operatorname{ord}(C)+\operatorname{ord}(h \circ \lambda) \leq \operatorname{ord}(u \circ \lambda) \\
\operatorname{ord}(C)+\operatorname{ord}(h \circ \lambda) \leq \operatorname{ord}(w \circ \lambda) \\
\operatorname{ord}(v \circ \lambda) \leq \operatorname{ord}(h \circ \lambda)
\end{array}\right.
$$

Note that we are not considering the second inequality in the case where $n=p$.

Let $\tilde{\lambda}$ be written as follows:

$$
\lambda_{i}(t)=a_{1}^{(i)} t^{\varepsilon_{1}(i)}+a_{2}^{(i)} t^{\varepsilon_{2}(i)}+\ldots,
$$

where $1 \leq \varepsilon_{1}(i)<\varepsilon_{2}(i)<\ldots$ and

$$
\left\{\begin{array}{ccc}
a_{1}^{(i)} \neq 0 & \text { if } & \lambda_{i}(t) \not \equiv 0 \\
\varepsilon_{1}(i)=\infty & \text { if } & \lambda_{i}(t) \equiv 0
\end{array}(1 \leq i \leq n),\right.
$$

$C(t)=u_{1} t^{b_{1}}+u_{2} t^{b_{2}}+\ldots$ where $1 \leq b_{1}<b_{2}<\ldots$ and $u_{1} \neq 0$. Since condition (3.1) is invariant under rotation, we can assume that $\varepsilon_{1}(1)<\varepsilon_{1}(i)$ for $i \neq 1$. Let $f_{j}(\lambda(t))=d_{1}^{(j)} t_{1}^{(j)}+d_{2}^{(j)} t_{2}^{(j)}+\ldots, \quad$ where $1 \leq$ $q_{1}^{(j)}<q_{2}^{(j)}<\ldots(1 \leq j \leq p)$. Then 


$$
\frac{d f_{j} \circ \lambda}{d t}(t)=q_{1}^{(j)} d_{1}^{(j)} t^{q_{1}^{(j)}-1}+q_{2}^{(j)} d_{2}^{(j)} t^{q_{2}^{(j)}-1}+\ldots
$$

$(1 \leq j \leq p)$. It follows from (3.3) that

(3.4) $q_{1}^{(j)} \geq \operatorname{ord}(C)+\operatorname{ord}(h \circ \lambda)$ for all $j \in\{1, \ldots, p\}$.

By (3.3) again, we have

$$
\begin{array}{r}
\varepsilon_{1}(1)+\operatorname{ord}\left(\sum_{1 \leq i_{1}<\ldots<i_{p} \leq n}\left|\operatorname{det}\left(\frac{D\left(f_{1}, \ldots, f_{p}\right)}{D\left(x_{i_{1}}, \ldots, x_{i_{p}}\right)}(\lambda(t))\right)\right|\right) \\
\leq \operatorname{ord}(h \circ \lambda) .
\end{array}
$$

Therefore there is a $p$-tuple of integers $\left(k_{1}, \ldots, k_{p}\right)$ with $1 \leq k_{1}<\cdots<k_{p} \leq n$ such that

$$
\begin{aligned}
& \operatorname{ord}\left(\left|\operatorname{det}\left(\frac{D\left(f_{1}, \ldots, f_{p}\right)}{D\left(x_{k_{1}}, \ldots, x_{k_{p}}\right)}(\lambda(t))\right)\right|\right) \\
& \quad \leq \operatorname{ord}\left(\left|\operatorname{det}\left(\frac{D\left(f_{1}, \ldots, f_{p}\right)}{D\left(x_{i_{1}}, \ldots, x_{i_{p}}\right)}(\lambda(t))\right)\right|\right) \\
& \quad \text { for any }\left(i_{1}, \ldots, i_{p}\right), \text { and } \\
& \operatorname{ord}\left(\left|\operatorname{det}\left(\frac{D\left(f_{1}, \ldots, f_{p}\right)}{D\left(x_{k_{1}}, \ldots, x_{k_{p}}\right)}(\lambda(t))\right)\right|\right) \\
& \quad \leq \operatorname{ord}(h \circ \lambda)-\varepsilon_{1}(1) .
\end{aligned}
$$

We continue the proof of the converse, dividing it into two cases. We first consider the case where $n>p$. Then we have the following

Claim. $k_{1}>1$.

Proof. Since

$$
\frac{d f_{j} \circ \lambda}{d t}(t)=\sum_{i=1}^{n} \frac{\partial f_{j}}{\partial x_{i}}(\lambda(t)) \frac{d \lambda_{i}}{d t}(t),(1 \leq j \leq p),
$$

we have

$$
\begin{aligned}
& \left(\begin{array}{c}
\frac{d f_{1} \circ \lambda}{d t}(t) \\
\vdots \\
\frac{d f_{p} \circ \lambda}{d t}(t)
\end{array}\right) \\
& =\frac{d \lambda_{1}}{d t}(t)\left(\begin{array}{c}
\frac{\partial f_{1}}{\partial x_{1}}(\lambda(t)) \\
\vdots \\
\frac{\partial f_{p}}{\partial x_{1}}(\lambda(t))
\end{array}\right)+\ldots+\frac{d \lambda_{n}}{d t}(t)\left(\begin{array}{c}
\frac{\partial f_{1}}{\partial x_{n}}(\lambda(t)) \\
\vdots \\
\frac{\partial f_{p}}{\partial x_{n}}(\lambda(t))
\end{array}\right) .
\end{aligned}
$$

Here we remark that, by (3.4)

$$
\begin{aligned}
& \operatorname{ord}\left(\frac{1}{\lambda_{1}^{\prime}(t)} \cdot \frac{d f_{j} \circ \lambda}{d t}(t)\right)=q_{1}^{(j)}-\varepsilon_{1}(1) \\
& \quad \geq \operatorname{ord}(C)+\operatorname{ord}(h \circ \lambda)-\varepsilon_{1}(1),
\end{aligned}
$$

$(1 \leq j \leq p)$, and

$$
\operatorname{ord}\left(\frac{\lambda_{i}^{\prime}(t)}{\lambda_{1}^{\prime}(t)}\right) \geq 1 \quad(2 \leq i \leq n) .
$$

Assume, by contradiction, that $k_{1}=1$ in (3.6). For simplicity, set

$$
A(t)=\left(\begin{array}{cccc}
\frac{\partial f_{1}}{\partial x_{1}}(\lambda(t)) & \frac{\partial f_{1}}{\partial x_{k_{2}}}(\lambda(t)) & \ldots & \frac{\partial f_{1}}{\partial x_{k_{p}}}(\lambda(t)) \\
\vdots & \vdots & & \vdots \\
\frac{\partial f_{p}}{\partial x_{1}}(\lambda(t)) & \frac{\partial f_{p}}{\partial x_{k_{2}}}(\lambda(t)) & \ldots & \frac{\partial f_{p}}{\partial x_{k_{p}}}(\lambda(t))
\end{array}\right) .
$$

Then the determinant of the matrix $A(t)$ is the summation of determinants of the following matrices:

$$
\begin{aligned}
& \left(\frac{1}{\lambda_{1}^{\prime}(t)}\left(\begin{array}{c}
\frac{d f_{1}>\lambda}{d t}(t) \\
\vdots \\
\frac{d f_{p} \lambda}{d t}(t)
\end{array}\right) \begin{array}{cccc}
\frac{\partial f_{1}}{\partial x_{k_{2}}}(\lambda(t)) & \ldots & \frac{\partial f_{1}}{\partial x_{k_{p}}}(\lambda(t)) \\
\vdots & & \vdots \\
\frac{\partial f_{p}}{\partial x_{k_{2}}}(\lambda(t)) & \ldots & \frac{\partial f_{p}}{\partial x_{k_{p}}}(\lambda(t))
\end{array}\right),
\end{aligned}
$$

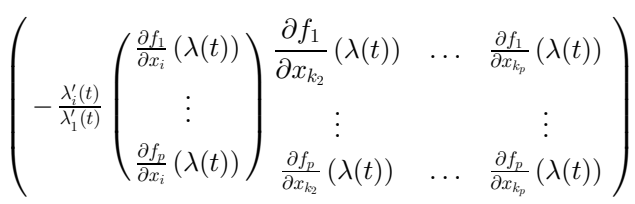

for $i \in\{2, \ldots, n\}$.

By (3.8) the order of the determinant of the matrix (3.10) is bigger than or equal to ord $(C)+$ $\operatorname{ord}(h \circ \lambda)-\varepsilon_{1}(1)$, and by (3.5) the order of the determinant of the matrix (3.11) is bigger than the order of the determinant of the matrix (3.10). Therefore we have

$$
\begin{aligned}
\operatorname{ord}(|\operatorname{det} A(t)|) & \geq \operatorname{ord}(C)+\operatorname{ord}(h \circ \lambda)-\varepsilon_{1}(1) \\
& >\operatorname{ord}(h \circ \lambda)-\varepsilon_{1}(1)
\end{aligned}
$$

which contradicts (3.6). This completes the proof of the claim.

It follows from the Claim that there is a $p$-tuple $\left(k_{1}, \ldots, k_{p}\right)$ with $1<k_{1}<\cdots<k_{p} \leq n$ such that condition (3.6) holds. Then

$$
\begin{aligned}
& \operatorname{ord}\left(\left|\operatorname{det}\left(\frac{D\left(f_{1}, \ldots, f_{p}, \rho\right)}{D\left(x_{1}, x_{k_{1}}, \ldots, x_{k_{p}}\right)}(\lambda(t))\right)\right|\right) \\
& \quad \leq \operatorname{ord}(\lambda)+\operatorname{ord}\left(\left|\operatorname{det}\left(\frac{D\left(f_{1}, \ldots, f_{p}\right)}{D\left(x_{k_{1}}, \ldots, x_{k_{p}}\right)}(\lambda(t))\right)\right|\right) \\
& \quad \leq \varepsilon_{1}(1)+\operatorname{ord}(h \circ \lambda)-\varepsilon_{1}(1)=\operatorname{ord}(h \circ \lambda) .
\end{aligned}
$$

This contradicts (3.3), and it follows that $K_{m}(f,.) \precsim T_{m}(f,$.$) .$ 
We next consider the case where $n=p$. Using a similar argument to the proof of the above Claim, we get the same contradiction for

$$
A(t)=\left(\begin{array}{cccc}
\frac{\partial f_{1}}{\partial x_{1}}(\lambda(t)) & \frac{\partial f_{1}}{\partial x_{2}}(\lambda(t)) & \ldots & \frac{\partial f_{1}}{\partial x_{n}}(\lambda(t)) \\
\vdots & \vdots & & \vdots \\
\frac{\partial f_{n}}{\partial x_{1}}(\lambda(t)) & \frac{\partial f_{n}}{\partial x_{2}}(\lambda(t)) & \ldots & \frac{\partial f_{n}}{\partial x_{n}}(\lambda(t))
\end{array}\right) .
$$

Therefore it follows that $K_{m}(f,.) \precsim T_{m}(f,$.$) , and$ this completes the proof.

Remark 3.2. The proof of Theorem 1.3 uses essentially the curve selection lemma. Hence it is not difficult to see that the results are valid if we suppose only that $f$ is an arc-analytic and differentiable subanalytic map-germ; see [10], [5] and [3] for the notions and properties of subanalytic and arc-analytic functions.

\section{Applications of our main result to the} relative case.

4.1. $\boldsymbol{\Sigma}$-r -compatibility. We now introduce some notion for a $C^{r}$-map germ $f:\left(\mathbf{R}^{n}, 0\right) \rightarrow\left(\mathbf{R}^{p}, 0\right)$ in order to extend to the relative case the previous equivalence defined in the non-relative case.

Let $\Sigma$ be a germ at $0 \in \mathbf{R}^{n}$ of closed set such that $0 \in \Sigma$. Given a map $g \in \mathcal{E}_{[r]}(n, p)$ with $j^{r} g(\Sigma ; 0)=j^{r} f(\Sigma ; 0)$, let $f_{t}:\left(\mathbf{R}^{n}, 0\right) \rightarrow\left(\mathbf{R}^{p}, 0\right)$ denote the $C^{r}$ mapping defined by

$$
f_{t}(x)=f(x)+t(g(x)-f(x)) \text { for } t \in[0,1] .
$$

Definition 4.1. A condition $(*)$ on a $C^{r}$ map $f$ is called $\Sigma$-r-compatible in the direction $g$, if $f_{t}$ satisfies condition $(*)$ for any $t \in[0,1]$. If condition $(*)$ is $\Sigma$-r-compatible in any direction $g \in \mathcal{E}_{[r]}(n, p)$ with $j^{r} g(\Sigma ; 0)=j^{r} f(\Sigma ; 0)$, we simply say condition $(*)$ is $\Sigma$-r-compatible.

Let $f:\left(\mathbf{R}^{n}, 0\right) \rightarrow\left(\mathbf{R}^{p}, 0\right)$ be a $C^{1}$ map-germ, $\Sigma \subset \mathbf{R}^{n}$ be a germ of closed set such that $0 \in \Sigma$ and $r \in \mathbf{N}$. For $m \in \mathbf{N}$, we introduce the following conditions:

$$
I_{r}^{T}(m): \exists c, \delta>0 \text { such that } T_{m}(f, x) \geq c(d(x, \Sigma))^{r m}
$$

for $\|x\|<\delta$,

$I_{r}^{K}(m): \exists c, \delta>0$ such that $K_{m}(f, x) \geq c(d(x, \Sigma))^{r m}$ for $\|x\|<\delta$.

Remark 4.2. If $f$ is $C^{\omega}$, we have from Theorem 1.3, for any $m \in \mathbf{N}$,

$$
I_{r}^{T}(m) \text { holds if and only if } I_{r}^{K}(m) \text { holds. }
$$

Proposition 4.3. The conditions $I_{r}^{T}(m)$ and $I_{r}^{K}(m)$ are r-compatible.

Proof. Let $f_{t}=f+t h$ with $h=g-f$. Then $\|h\|=o\left(d(., \Sigma)^{r}\right),\left\|f_{t}\right\| \geq\|f\|-\|h\|$ and the expansion of the determinants give

$$
T_{m}\left(f_{t}, x\right)=T_{m}(f, x)+o(d(x, \Sigma))^{r m}
$$

and

$$
K_{m}\left(f_{t}, x\right)=K_{m}(f, x)+o(d(x, \Sigma))^{r m} .
$$

Thus the $r$-compatibilities of $I_{r}^{T}(m)$ and $I_{r}^{K}(m)$ follow.

As a corollary of Theorem 1.3, we have the following result.

Corollary 4.4. Let $\Sigma$ be a germ at $0 \in \mathbf{R}^{n}$ of closed set such that $0 \in \Sigma$. Let $r \in \mathbf{N}$, and let $f \in$ $\mathcal{E}_{[r]}(n, p), n \geq p$. Suppose that $j^{r} f(\Sigma, 0)$ has a $C^{\omega}$ realisation. Then for any $m \in \mathbf{N}$,

\section{$I_{r}^{T}(m)$ holds if and only if $I_{r}^{K}(m)$ holds.}

Proof. Let $g:\left(\mathbf{R}^{n}, 0\right) \rightarrow\left(\mathbf{R}^{p}, 0\right)$ be a $C^{\omega}$ realisation of $j^{r} f(\Sigma, 0)$. From Theorem 1.3, conditions $I_{r}^{T}(m)$ and $I_{r}^{K}(m)$ are equivalent for $g$. Now, by Proposition 4.3, the result follows.

Remark 4.5. As pointed out in [2], any $r$-jet, $r \in \mathbf{N}$, has a unique polynomial realisation of degree not exceeding $r$ in the non-relative case, but some $r$-jets do not have even a $C^{\omega}$ realisation in the general relative case. Therefore, in the above theorem, the assumption that $j^{r} f(\Sigma, 0)$ has a $C^{\omega}$ realisation makes sense.

Lemma 4.6. For $X_{1}, \ldots, X_{l} \geq 0$ and a positive integer $m \in \mathbf{N}$, we have

$$
\left(X_{1}+\ldots+X_{l}\right)^{m} \approx X_{1}^{m}+\ldots+X_{l}^{m} .
$$

Therefore we see that $K_{1} \approx T_{1}$ if and only if for any $m \in \mathbf{N}, K_{m} \approx T_{m}$.

As a corollary of Lemma 4.6 and Corollary 4.4, we have the following result.

Corollary 4.7. Let $\Sigma$ be a germ at 0 of closed set. Let $r \in \mathbf{N}$, and let $f \in \mathcal{E}_{[r]}(n, p), n \geq p$. Suppose that $j^{r} f(\Sigma, 0)$ has a $C^{\omega}$ realisation. Then the following conditions are equivalent:

(1) There exists $m \in \mathbf{N}$ such that $I_{r}^{T}(m)$ holds,

(2) For all $m \in \mathbf{N}, I_{r}^{T}(m)$ holds,

(3) There exists $m \in \mathbf{N}$ such that $I_{r}^{K}(m)$ holds,

(4) For all $m \in \mathbf{N}, I_{r}^{K}(m)$ holds.

Remark 4.8. It follows from the proof of Theorem 1.3 that the equivalence between conditions $T_{m}$ and $K_{m}$ holds for any $C^{1}$ map $f$ in a 
category where the analytic curve selection lemma is valid.

4.2. Characterisations of finite $\Sigma-S V$ determinacy. Let $\mathcal{E}(n)^{p}, n>p$, be the set of $C^{\infty}$ map-germs: $\mathbf{R}^{n} \rightarrow \mathbf{R}^{p}$ at $0 \in \mathbf{R}^{n}$, and let $\Sigma$ be a germ of closed subset of $\mathbf{R}^{n}$ such that $0 \in \Sigma$. We say that $f, g \in \mathcal{E}_{[s]}(n, p)$ are $\Sigma$-SV-equivalent if there is a local homeomorphism $\varphi:\left(\mathbf{R}^{n}, 0\right) \rightarrow\left(\mathbf{R}^{n}, 0\right)$ fixing $\Sigma$ such that $\varphi\left(f^{-1}(0)\right)=g^{-1}(0)$, and $f, g \in \mathcal{E}_{[s]}(n, p)$ are $\Sigma$-V-equivalent if $f^{-1}(0)$ is homeomorphic to $g^{-1}(0)$ as germs at $0 \in \mathbf{R}^{n}$ by a homeomorphism which fixes $f^{-1}(0) \cap \Sigma$. Then $f \in \mathcal{E}(n)^{p}$ is called finitely $\Sigma$-SV-determined (resp. finitely $\Sigma$ - $V$ determined) if there is a positive integer $k$ such that for any $g \in \mathcal{E}(n)^{p}$ with $j^{k} g(\Sigma ; 0)=j^{k} f(\Sigma ; 0), g$ is $\Sigma$-SV-equivalent (resp. $\Sigma$ - $V$-equivalent) to $f$.

Let $\varphi=\left(\varphi_{1}, \ldots, \varphi_{p}\right): \mathbf{R}^{n} \rightarrow \mathbf{R}^{p}, n>p$, be a $C^{\infty}$ map-germ at $0 \in \mathbf{R}^{n}$. We denote by $I_{K}(\varphi)$ the ideal of $\mathcal{E}(n)$ generated by $\varphi_{1}, \ldots, \varphi_{p}$ and the Jacobian determinants

$$
\frac{D\left(\varphi_{1}, \ldots, \varphi_{p}\right)}{D\left(x_{i_{1}}, \ldots, x_{i_{p}}\right)}(x),\left(1 \leq i_{1}<\ldots<i_{p} \leq n\right) .
$$

We define also the ideal of $\mathcal{E}(n)$, denoted by $I_{T}(\varphi)$, generated by $\varphi_{1}, \ldots, \varphi_{p}$ and the Jacobian determinants

$$
\frac{D\left(\varphi_{1}, \ldots, \varphi_{p}, \rho\right)}{D\left(x_{i_{1}}, \ldots, x_{i_{p+1}}\right)}(x),\left(1 \leq i_{1}<\ldots<i_{p+1} \leq n\right)
$$

where $\rho(x)=\|x\|^{2}$.

Let $\mathfrak{m}_{\Sigma}^{\infty}$ be the ideal of $\mathcal{E}(n)$ consisting of germs $f$ such that $j^{\infty} f(x)=0$ for all $x \in \Sigma$, namely $\mathfrak{m}_{\Sigma}^{\infty}=$ $\left\{f \in \mathcal{E}(n): j^{\infty} f(\Sigma ; 0)=0\right\}$.

Definition 4.9. Let $I$ be an ideal of $\mathcal{E}(n)$. We say that $I$ is $\Sigma$-elliptic if there is $f \in I$ such that $|f(x)| \geq C d(x, \Sigma)^{\alpha}$ in a neighbourhood of 0 , where $C$ and $\alpha$ are positive constants.

A germ of closed subset $\Sigma$ of $\mathbf{R}^{n}$ is called coherent if $\mathfrak{m}_{\Sigma}$ is a finitely generated ideal of $\mathcal{E}(n)$. Then we have the following characterisations of finite $\Sigma$ - $S V$-determinacy.

Theorem 4.10. ([2], Theorem 5.7). Let $\Sigma$ be a coherent germ of closed subset of $\mathbf{R}^{n}$ such that $0 \in \Sigma$. Then the following conditions are equivalent for $\varphi \in \mathcal{E}(n)^{p}$ where $n>p$ :
(1) $\varphi$ is finitely $\Sigma$-SV-determined.

(2) $\varphi$ is finitely $\Sigma$-V-determined.

(3) $I_{K}(\varphi)$ is $\Sigma$-elliptic.

(4) $\mathfrak{m}_{\Sigma}^{\infty} \subset I_{K}(\varphi)$.

If moreover $\varphi$ is analytic, they are also equivalent to:

(5) $\mathfrak{m}_{\Sigma}^{\infty} \subset I_{T}(\varphi)$.

Theorem 1.3 takes an important role in the proof of the above theorem. For the detailed proof and more characterisations of finite $\Sigma$-SVdeterminacy, see $\S 5$ in [2].

Acknowledgements. This research is partially supported by JSPS KAKENHI Grant Numbers JP26287011, JP20K03611, and HUTE ShortTerm Fellowship Program 2016.

\section{References}

[ 1 ] K. Bekka and S. Koike, The Kuo condition, an inequality of Thom's type and $(C)$-regularity, Topology 37 (1998), no. 1, 45-62.

[ 2 ] K. Bekka and S. Koike, Characterisations of Vsufficiency and $C^{0}$-sufficiency of relative jets, arXiv.1703.07069v5.

[ 3 ] E. Bierstone and P. D. Milman, Arc-analytic functions, Invent. Math. 101 (1990), no. 2, 411424.

[ 4 ] J. Bochnak and S. Łojasiewicz, A converse of the Kuiper-Kuo theorem, in Proceedings of Liverpool Singularities-Symposium, I (1969/700), 254-261. Lecture Notes in Math., 192, Springer, Berlin, 1971.

[ 5 ] H. Hironaka, Subanalytic sets, in Number theory, algebraic geometry and commutative algebra, in honor of Yasuo Akizuki, Kinokuniya, Tokyo, 1973 , pp. $453-493$.

[6 ] N. H. Kuiper, $C^{1}$-equivalence of functions near isolated critical points, in Symposium on Infinite-Dimensional Topology (Louisiana State Univ., Baton Rouge, La., 1967), 199-218, Ann. of Math. Studies, 69, Princeton Univ. Press, Princeton, NJ, 1972.

[ 7 ] T. C. Kuo, On $C^{0}$-sufficiency of jets of potential functions, Topology 8 (1969), 167-171.

[ 8 ] T. C. Kuo, A complete determination of $C^{0}$-sufficiency in $J^{r}(2,1)$, Invent. Math. 8 (1969), 226235.

[9] T. C. Kuo, Characterizations of $v$-sufficiency of jets, Topology 11 (1972), 115-131.

[ 10 ] K. Kurdyka, Ensembles semi-algébriques symétriques par arcs, Math. Ann. 282 (1988), no. 3, 445-462. 